\title{
Связь длинноволновой границы чувствительности пленок PbSnTe : In, полученных методом молекулярно-лучевой эпитаксии, с их составом и структурой
}

\author{
(C) А.Н. Акимов ${ }^{1}$, А.Э. Климов ${ }^{1,2, \uparrow, ~ Н . С . ~ П а щ и н ~}{ }^{1}$, А.С. Ярошевич ${ }^{1}$, \\ М.Л. Савченко ${ }^{1}$, В.С. Эпов ${ }^{1}$, Е.В. Федосенко ${ }^{1}$ \\ ${ }^{1}$ Институт фризики полупроводников Сибирского отделения Российской академии наук, \\ 630090 Новосибирск, Россия \\ ${ }^{2}$ Новосибирский Государственный Технический Университет, \\ 630073, Новосибирск, Россия \\ ฯ E-mail: klimov@isp.nsc.ru
}

(Получена 27 апреля 2017 г. Принята к печати 12 мая 2017 г.)

\begin{abstract}
Исследованы пленки $\mathrm{PbSnTe}:$ In с длинноволновой границей чувствительности свыше 20 мкм и низкой проводимостью без освещения. Проведено сравнение спектральных зависимостей фотопроводимости, полученных при различных температурах с использованием фурье-спектрометра, с составом пленок, определенным методом рентгеновского микроанализа. Обнаружена немонотонная зависимость длинноволнового края фоточувствительности от температуры, которая объясняется комбинацией температурной зависимости ширины запрещенной зоны PbSnTe и эффекта Бурштейна-Мосса, дающего наибольший вклад в измерения при низких температурах из-за большого времени жизни неравновесных носителей заряда. Показано, что различия в значениях ширины запрещенной зоны, определяемых из измеренного состава и температурных зависимостей длинноволнового края чувствительности, могут быть связаны с неоднородностью состава пленок, получаемых методом молекулярно-лучевой эпитаксии.
\end{abstract}

DOI: 10.21883/FTP.2017.11.45114.29

\section{1. Введение}

Ширина запрещенной зоны твердого раствора $\mathrm{Pb}_{1-x} \mathrm{Sn}_{x}$ Те уменьшается от $E_{g}=0.19$ эВ $(x=0)$ до нуля в точке инверсии зон при $x=0.35(T=0)[1,2]$. Сколь угодно малая $E_{g}$ вблизи $x=0.35$ делает $\mathrm{PbSnTe}$ привлекательным для создания фотоприемников (ФП) сверхдальнего инфракрасного ИК (свыше 14-20 мкм) и терагецового (свыше 100 мкм) диапазонов на основе межзонных оптических переходов. Многоэлементные фотоприемные устройства (ФПУ) диапазона $\lambda_{\text {red }} \leq 20-25$ мкм на основе пленок PbSnTe c рекордными для рабочей $T \approx 7 \mathrm{~K}$ значениями мощности, эквивалентной шуму (noice-equivalent power), $\mathrm{NEP} \leq 10^{-18} \mathrm{BT} / \Gamma_{\coprod^{0.5}}$, были реализованы в [3]. При этом необходимые для функционирования ФПУ низкие темновые токи ФП были достигнуты за счет легирования пленок $\mathrm{PbSnTe}$ индием.

Проблема продвижения ФПУ этого типа в более длинноволновой диапазон связана с тем, что с помощью легирования In темновая проводимость $\mathrm{PbSnTe}$ может быть снижена до требуемой величины только в определенной области составов - не более чем до $x \sim 0.28$ [4,5]. Согласно [2], это соответствует $E_{g} \leq 0.038$ эВ и $\lambda_{\text {red }} \leq 32$ мкм. Однако достаточно низкую проводимость при $T \leq 20 \mathrm{~K}$ имеют и отдельные пленки $\mathrm{PbSnTe}: \mathrm{In}$, получаемые методом молекулярно-лучевой эпитаксии (МЛЭ) [6], с составом вплоть до $x \sim 0.33$, которому соответствует расчетная $\lambda_{\text {red }} \sim 115$ мкм. Вместе с тем оценки $\lambda_{\text {red }}$, полученные с использованием фурье-спектрометра [7] и низкотемпературных излучателей типа ,абсолютно черное тело“ $\left(T_{b b}<40-80 \mathrm{~K}\right)$, показали, что у исследованных образцов $\lambda_{\text {red }} \leq 30-40$ мкм. Одной из причин такого значительного расхождения может быть то, что зависимость $E_{g}(x)[2]$ требует корректировки для PbSnTe : In с содержанием In до нескольких атомных процентов, представляющего собой по сути четверное, а не тройное соединение. Другой возможной причиной является неоднородность состава [8]. Неоднородность состава пленок PbSnTe : In как по толщине, так и по площади описана в [9], ее возможная роль в появлении гигантской отрицательной фотопроводимости отмечена в [7].

Цель данной работы - исследование особенностей спектров фотопроводимости пленок $\mathrm{PbSnTe}$ : In при различной температуре, определение на их основе состава пленок с низкой темновой проводимостью и сравнение еe с данными, получаемыми методом рентгеновского микроанализа.

\section{2. Образцы и методика эксперимента}

Исследовались полученные методом МЛЭ монокристаллические пленки $\mathrm{Pb}_{1-x} \mathrm{Sn}_{x} \mathrm{Te}: \mathrm{In} / \mathrm{BaF}_{2}$ микронной толщины с составами до $x \sim 0.34$, переходящие при $T<20 \mathrm{~K}$ в высокоомное состояние. Некоторые особенности технологии получения изложены в [9]. Кристаллическое совершенство пленок подтверждается как данными дифракции быстрых электронов на отражение, так и высокой подвижностью носителей заряда 
при низких (гелиевых) температурах. Для исследований выбирались пленки с максимальной подвижностью не менее $10^{4} \mathrm{~cm}^{2} / \mathrm{B} \cdot \mathrm{c}$, которая обычно наблюдается в области температур 20-30 K. Концентрация и подвижность носителей заряда определялись из измерений эффекта Холла в магнитном поле $B=0.22$ Тл. При измерениях эффекта Холла и температурной зависимости тока (проводимости) образцы размещались в металлической камере, экранированной от фонового излучения, непосредственно в парах гелия или в жидком гелии. Для контроля фоточувствительности образцов в области фундаментального поглощения в той же измерительной камере размещались источники излучения: миниатюрная лампа накаливания с вакуумированной стеклянной колбой (край пропускания около 3.5 мкм) и светодиод видимого диапазона спектра. Измерения спектральной зависимости фотопроводимости при разных температу-

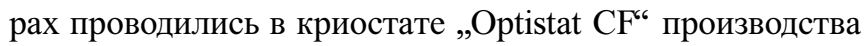
фирмы Oxford Instruments. Криостат охлаждался постоянным потоком жидкого гелия. Образцы в рабочую камеру загружались сверху на подвижном держателе. В криостате были установлены полипропиленовые окна, имеющие относительно высокое пропускание $(\sim 90-95 \%)$ в диапазоне 13-1000 мкм. Криостат смонтирован в рабочем объеме инфракрасного фурье-спектрометра Bruker Vertex 80v. На вход усилителя спектрометра подавался сигнал не с фотоприемника спектрометра, а с выхода широкополосного усилителя напряжения, к которому был подключен исследуемый образец. При измерении фотопроводимости изучаемая система последовательно соединялась с нагрузочным резистором, сопротивление которого для каждой температуры выбиралось примерно равным сопротивлению исследуемой структуры. Схема замыкалась источником постоянного напряжения (до 9В), измеряемое напряжение снималось с сопротивления нагрузки, далее производилась фильтрация сигнала по частоте, его усиление и подача на АЦП спектрометра.

Так как при такой методике измерений суммарное освещение образца фоновым ИК излучением и излучением фурье-спектрометра велико, сопротивление образцов и соответственно величины выбираемой нагрузки менялись согласованно, с максимальными значениями в области температуры примерно $20-30 \mathrm{~K}$.

\section{3. Экспериментальные результаты}

На рис. 1 приведены зависимости темнового тока двух образцов от обратной температуры. Напряжение на образцах $U=1.5 \mathrm{~B}$. Состав определен по данным рентгеновского микроанализа. Толщина образца с составом $x=0.235, d=0.6$ мкм (образец 1). Толщина образца с составом $x=0.306, d=1.7$ мкм (образец 2). В области температур $T<30 \mathrm{~K}$ образцы имели высокую фоточувствительность с увеличением тока при $T=4.2 \mathrm{~K}$ до более чем $4 \cdot 10^{-4} \mathrm{~A}$.

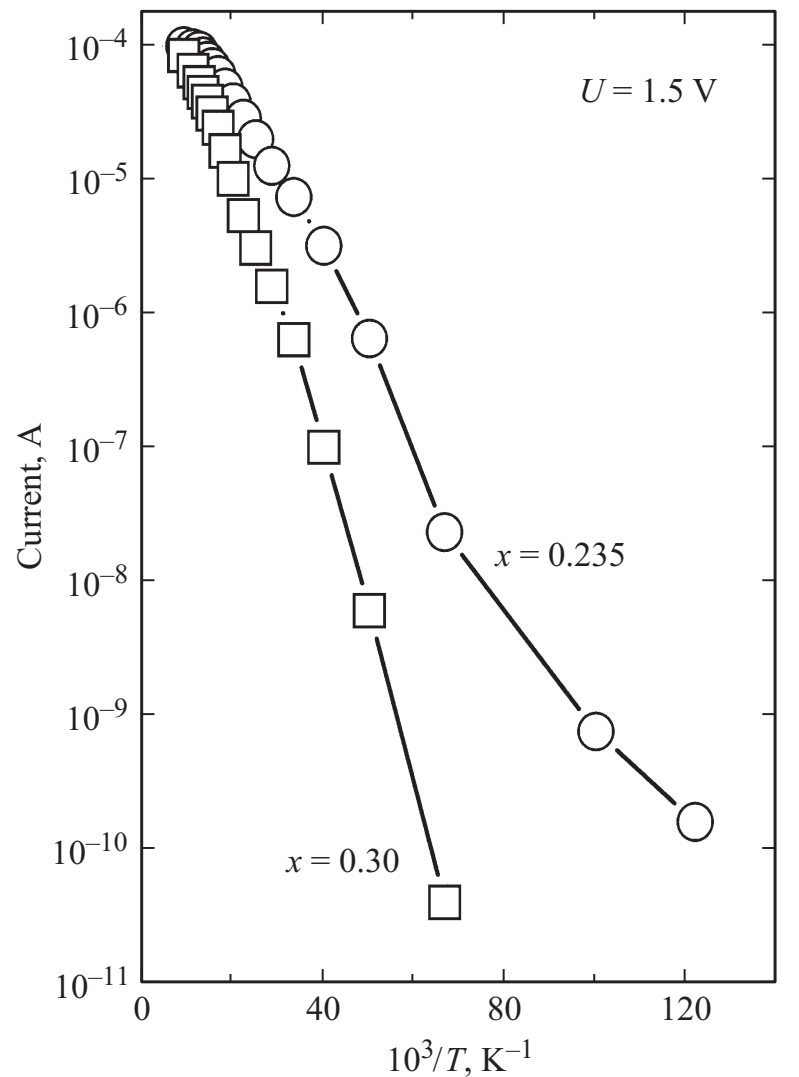

Рис. 1. Зависимости темнового тока образцов $1(x=0.235)$ и $2(x=0.30)$ от обратной температуры.

На рис. 2, $a$ приведены три спектра из серии спектров фотопроводимости образца 1 , измеренной при различных температурах с использованием $\mathrm{KBr}$-светоделителя, обеспечивающего спектральный диапазон измерений $350-10000 \mathrm{~cm}^{-1}(\sim 1-30$ мкм). Для удобства сравнения спектры нормированы на значения фотосигнала для каждой кривой при длине волны $\lambda=10.7$ мкм. Температура измерений показана на графике. Серия особенностей в виде глубоких провалов в области длин волн примерно 10-14 мкм связана с характеристиками (спектрами пропускания/отражения) окон криостата и оптических элементов фурье-спектрометра. Наблюдаемое увеличение значений на кривых в области длин волн выше примерно 20 мкм связано с тем, что характеристики использованного в этой серии измерений светоделителя не являются оптимальными для измерений в этом спектральном диапазоне (свыше 20 мкм). Это ведет к ухудшению отношения сигнал/шум и ошибкам при фурье-преобразовании, которые и приводят к появлению „хвостов“ („артефактов“) в длинноволновой области спектра. Другими словами, измерить по данной методике спектр фотопроводимости во всей предполагаемой области чувствительности пленок PbSnTe : In с одним и тем же светоделителем не представляется возможным. Три из серии спектральных зависимостей фотопроводимости в более длинноволновой области, измеренной для того 

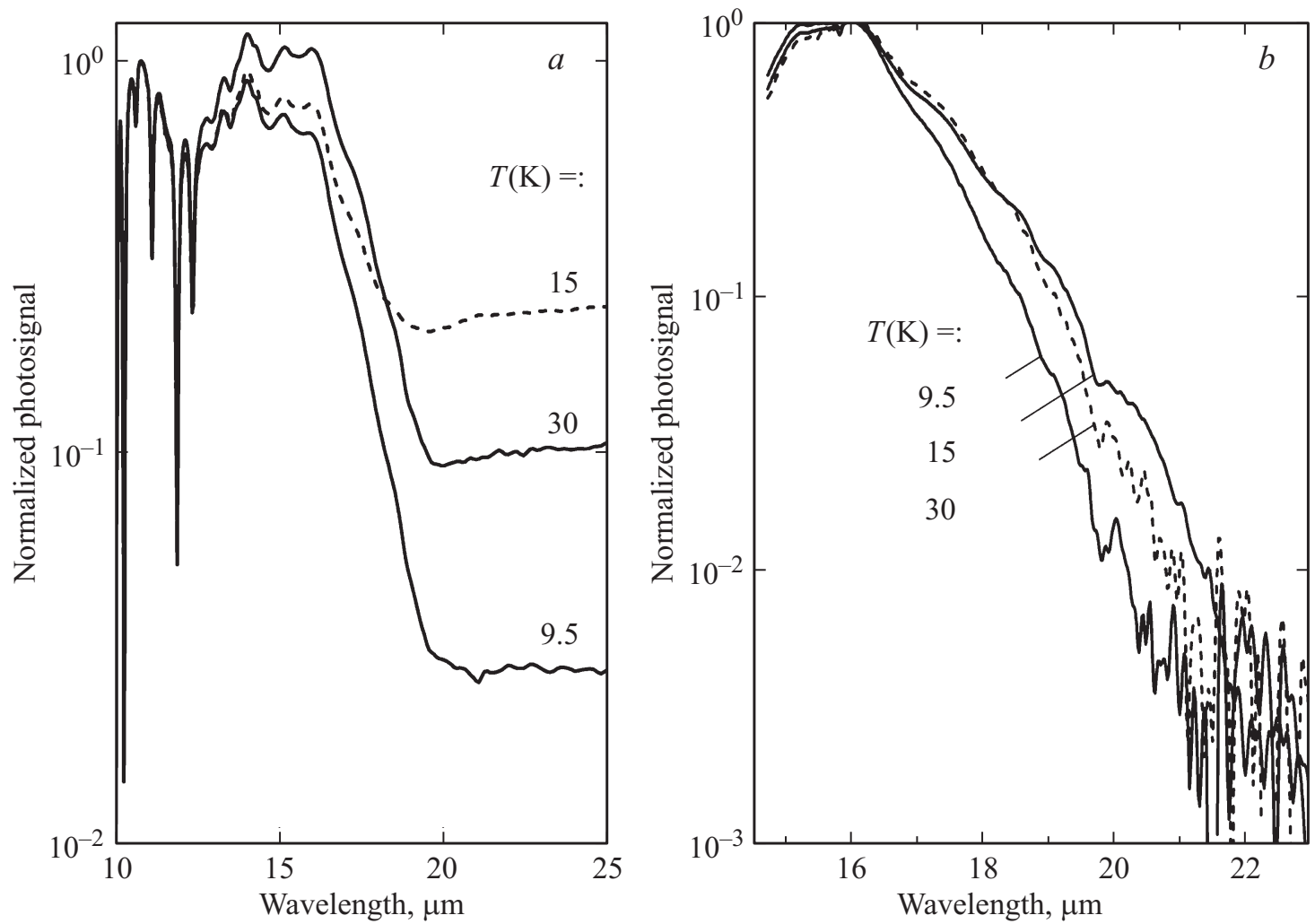

Рис. 2. $a$ - серия спектров фотопроводимости образца 1, измеренная при различных температурах с использованием $\mathrm{KBr}-$ светоделителя. $b$ - серия спектров фотопроводимости образца 1 , измеренная при различных температурах с использованием многослойного (multilayer) светоделителя. С данным светоделителем измерения при $\lambda<15$ мкм невозможны.

же образца с использованием многослойного (multilayer) светоделителя $\left(\sim 30-680 \mathrm{~cm}^{-1}\right)$ приведены на рис. $2, b$. В данном случае нормировка спектров сделана вблизи длин волн 15-16 мкм. Видно, что в данном случае „артефакты“ в длинноволновой области отсутствуют, и величина фотосигнала в области длин волн 16-22 мкм при всех использованных температурах измерений постепенно уменьшается с увеличением длины волны.

Аналогичная серия измерений спектра фотопроводимости была выполнена и на образце 2. В этом случае соотношение сигнала и шума было несколько хуже, чем для образца 1. При этом в области температуры $T=22-26 \mathrm{~K}$ красная граница чувствительности по уровню 0.01 от максимума не превышала $\lambda=24.5-25.7$ мкм.

\section{4. Обсуждение результатов}

Как было отмечено в предыдущем разделе, спектры фотопроводимости, измеренные с использованием фурье-спектрометра, имеют ряд особенностей, связанных с оптическими характеристиками спектрометра и используемого криостата, а также со спектральными характеристиками источника излучения спектрометра. В принципе от таких особенностей можно было бы избавиться путем нормировки полученных спектров на спектральную характеристику некого „эталонного“ фотоприемника (ФП), если бы имелась возможность установить такой ФП на место исследуемого образца. „Эталонный“ означает ФП с той же или более широкой спектральной областью чувствительности, в которой исследовались образцы PbSnTe : In. Желательно, чтобы спектральная характеристика эталонного ФП была измерена при той же температуре, что и спектры PbSnTe: In, так как характеристики „холодных“ окон криостата могут меняться с температурой. На практике реализация такой процедуры практически невозможна. Вместе с тем в рамках определенных предположений для достижений цели данной работы не обязательно знание нормированного спектра фотопроводимости во всей области фундаментального поглощения PbSnTe : In. А именно, если полагать, что максимальные длины волн, на которых наблюдается фотосигнал на рис. $2, b$, соответствуют межзонным оптическим переходам с энергией квантов $h v \approx E_{g}$ и не связаны, например, с переходами на уровни (или с уровней) в запрещенной зоне. Анализ серии спектров, три из которых показаны на рис. 2, b, с целью получения температурной зависимости края поглощения, был сделан для уровня сигнала 0.01 от максимального и представлен на рис. 3. Температура измерений $T=9.5,12,15,20,25$ и $30 \mathrm{~K}$. Там же для сравнения приведены температурные зависимости „края 


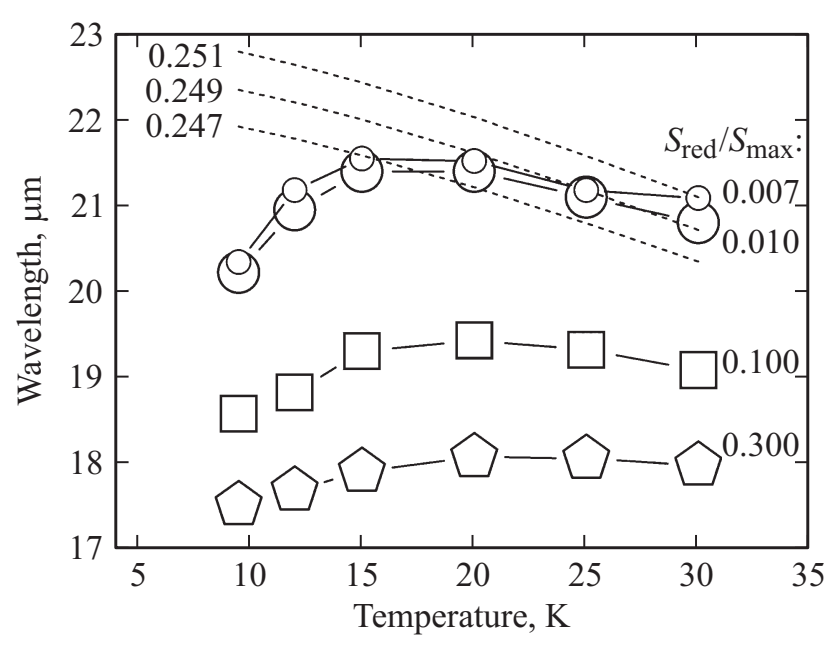

Рис. 3. Температурная зависимость края чувствительности образца 1, рассчитанная на основе рис. 2, $b$. Уровень сигнала, по которому определялся край чувствительности, указан возле соответствующих кривых справа. Пунктирными кривыми показаны расчетные зависимости, найденные с использованием выражения (1) для составов $x=0.247,0.249$ и 0.251 .
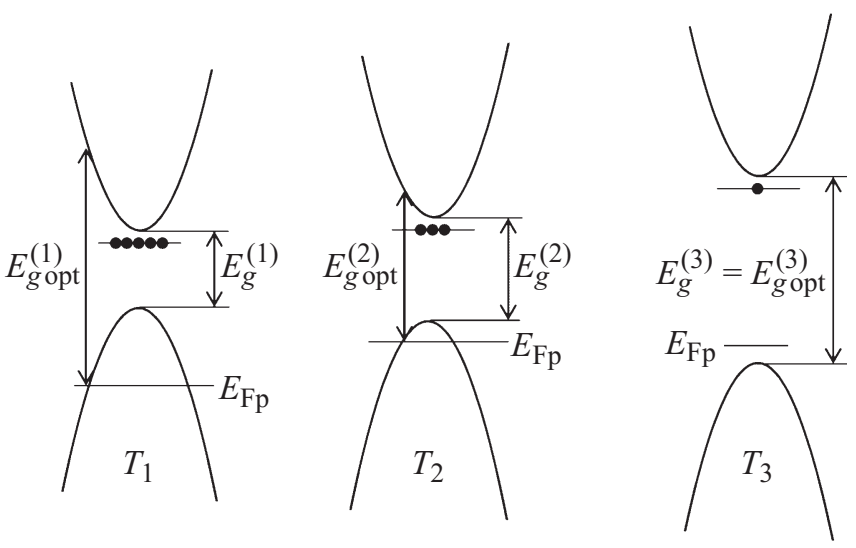

Pис. 4. Схематичная модель изменения ширины запрещенной зоны $E_{g}^{(i)}$ и „оптической“ ширины запрещенной зоны $E_{\mathrm{gopt}}^{(i)}$ в PbSnTe: In. Температура увеличивается от $i=1$ до $i=3$. $E_{\mathrm{F} p}-$ квазиуровень Ферми для дырок, определяющих величину проводимости. Захваченные на уровень ловушек электроны обозначены черными кружками.

поглощения“", сделанные по уровню сигнала 0.1 и 0.3 от максимального, а также по уровню 0.007 . Как видно из рисунка, на слабо отличающихся зависимостях по уровню 0.007 и 0.01 имеется выраженный максимум в области $T \sim 20-25 \mathrm{~K}$. Для уровней сигнала 0.1 и 0.3 этот максимум менее выражен, хотя также наблюдается.

В работе [10] приведены экспериментальные результаты зависимости $E_{\text {gopt }}$ от концентрации свободных носителей заряда. Оценка, проведенная на основании результатов этой работы, показала, что сдвиг $E_{\mathrm{gopt}}$, вызванный эффектом Мосc-Бурштейна, при концентрации носителей $\sim 10^{16} \mathrm{~cm}^{-3}$ может достигать 0.01 эВ.
Схема модели появления максимума, обусловленного эффектом Бурштейна-Мосса, представлена на рис. 4. При низких температурах (на схеме $T_{1}$ и $T_{2}$ ) сильное фоновое освещение и захват одного из типов неравновесных носителей заряда на уровни ловушек с большими временами жизни приводит к увеличению проводимости образцов до 6 порядков и более. На схеме для определенности без умаления общности показан только один уровень захвата для электронов. Большая концентрация дырок, по оценкам - выше $10^{16} \mathrm{~cm}^{-3}$, ведет к вырождению с расположением квазиуровня Ферми в валентной зоне. При этом оптическая ширина запрещенной зоны $E_{\text {gopt }}$ может быть существенно больше $E_{g}$. Соответственно край чувствительности сдвигается в область коротких длин волн. С увеличением температуры (индекс 2 на рисунке) величина сдвига уменьшается вследствие уменьшения времени жизни неравновесных носителей заряда и их концентрации. Одновременно с этим увеличивается и величина $E_{g}$, зависимость которой от состава и температуры имеет вид [2]

$$
E_{g}(x, T),{ }^{\ni} \mathrm{B}=0.19-0.543 x+4.5 \cdot 10^{-4} \cdot T^{2}(T+50)^{-1} .
$$

Область температур между индексами 1 и 2 соответствует на рис. 3 примерно $T \sim 10-20 \mathrm{~K}$. Дальнейшее увеличение температуры (индекс 3 на рис. 4) приводит к еще более сильному уменьшению времени жизни и концентрации неравновесных дырок, определяющих величину фотосигнала. При этом образец может быть и невырожденным, когда $E_{\text {gopt }}=E_{g}$. На рис. 3 приведены также расчетные зависимости края чувствительности в соответствии с выражением (1). Видно, что экспериментальные результаты хорошо совпадают с расчетом для значения $x=0.249 \pm 0.002$ в области $T>20 \mathrm{~K}$, где влияние эффекта Бурштейна-Мосса становится незначительным. Полученное из анализа спектров значение $x$ несколько больше измеренного методом рентгеновского микроанализа $x=0.235$.

Для второго образца выражение (1) и полученная из спектров фотопроводимости граница чувствительности $\lambda=24.5-25.7$ мкм при $T=22-26 \mathrm{~K}$ дает значение состава $x \sim 0.266 \pm 0.003$, что в отличие от образца 2 существенно меньше значения, полученного методом рентгеновского микроанализа $x=0.306$.

Таким образом, значения состава, полученные разными методами, удовлетворительно согласуются для образца 1 и существенно отличаются для образца 2. Одной из причин таких расхождений, с нашей точки зрения, может быть заметная неоднородность пленок по составу, как по толщине, так и по площади (см., например, [9]). Можно также осторожно предположить, что в области сравнительно больших значений $x$ (близко к 0.3 и более) и значительном (до нескольких атомных процентов) содержании индия выражение (1) не вполне справедливо. А именно оно было получено на основе исследований нелегированного PbSnTe, в то время как 
в случае $\mathrm{PbSnTe}:$ In речь может идти по существу не о тройном, а о четверном соединении.

\section{5. Заключение}

В работе выполнены исследования температурной зависимости края фотопроводимости образцов пленок $\mathrm{PbSnTe}: \mathrm{In}$, полученных методом молекулярно-лучевой эпитаксии, и на их основе сделаны расчеты состава пленок, которые сравнены с данными, полученными методом рентгеновского микроанализа. Расхождения полученных значений более существенны в области составов, приближающихся к бесщелевому $(x \sim 0.3$ и более). Представляется, что причиной такого расхождения может быть как неоднородность пленок по составу, так и большая концентрация индия, влияние которой на оптические характеристики может возрастать при увеличении $x$.

Работа выполнена при поддержке РФФИ, проект № 17-02-00575.

\section{Список литературы}

[1] J.O. Dimmock, I. Melngailis, A.J. Strauss. Phys. Rev. Lett., 16, 1193 (1966).

[2] W.W. Anderson. IEEE J. Quant. Electron., QE-13, 532 (1977).

[3] А.Э. Климов, В.Н. Шумский. В кн.: Матричные фботоприемные устройства инбракрасного диапазона, Новосибирск, Наука, 2001) гл. 6, с. 308.

[4] Б.А. Акимов, Л.И. Рябова, О.Б. Яценко, С.М. Чудинов. ФТП, 13, 752 (1979).

[5] S. Takaoka, T. Itoga, K. Murase. Jpn. J. Appl. Phys., 23, 216 (1984).

[6] А.Н. Акимов, Д.В. Ищенко, А.Э. Климов, И.Г. Неизвестный, И.С. Пащин, В.Н. Шерстякова, В.Н. Шумский. Микроэлектроника, 42, 83 (2013).

[7] А.Н. Акимов, А.Э. Климов, С.В. Морозов, С.П. Супрун, В.С. Эпов, И.В. Иконников, М.А. Фадеев, В.В. Румянцев. ФТП, 50, 1713 (2016).

[8] Г.А. Калюжная, К.В. Киселева. Тр. ФИАН, 177, 5 (1987).

[9] A.E. Klimov, D.V. Krivopalov, I.G. Neizvestnyi, V.N. Shumsky, N.I. Petikov, M.A. Torlin, E.V. Fedosenko. Appl. Surf. Sci., 78, 413 (1994).

[10] M.A. Rafea, F.S. Terra, M. Mounir, R. Labusch. Chalc. Lett., 6, 115 (2009)

Редактор Г.А. Оганесян

\section{Long-wavelegth limit of MBE-grown PbSnTe : In films sensitivity: relation to structure and composition}

\author{
A.N. Akimov ${ }^{1}$, A.E. Klimov ${ }^{1,2}$, N.S. Paschin ${ }^{1}$, \\ A.S. Yaroshevich ${ }^{1}$, M.L. Savchenko ${ }^{1}$, \\ V.S. Epov ${ }^{1}$, E.V. Fedosenko ${ }^{1}$ \\ ${ }^{1}$ Institute of Semiconductor Physics, \\ Russian Academy of Sciences, Siberian Branch, \\ 630090 Novosibirsk, Russia \\ ${ }^{2}$ Novosibirsk State Technical University, \\ 630073 Novosibirsk, Russia
}

\begin{abstract}
In the present work PbSnTe films doped with In and featuring the long-wavelength limit of sensitivity above 20 micron and low dark conductivity have been studied. Spectral characteristics of photoconductivity measured using Fourier spectrometer have been compared to the $X$-Ray microanalysis data on film composition. It has been found that the long-wavelegth limit of sensitivity non-monotonically depended on temperature. Such behaviour can be explained by a combined influence of the band gap thermal dependence and the Burstein-Moss effect, which mostly contributes during the low-temperature measurements because of the extremely long lifetime of the nonequilibrium carriers. It has been shown that the significant difference between band gap values calculated from the measured composition or from the optical measurements of the long-wavelegth cutoff can be due to inhomogeneous structure and nonuniform composition of MBE-grown films.
\end{abstract}

